\title{
How Person-Supervisor Fit Influence the Work Engagement of New Hires?
}

\author{
Yuanyuan Wang \\ Department of Teacher Education, Weifang University, Shandong, China \\ lilywangyuan@126.com
}

\begin{abstract}
For new hires, receiving support from their supervisor is critical for familiarizing themselves with their job and engaging it as soon as possible. The aim of this paper is to explore the influencing mechanism of person-supervisor fit(PSF) on the work engagement (WE) of new hires as well as the mediating role of person-organization fit(POF) and person-job fit(PJF). Five hundred new hires were selected through cluster sampling, and asked to fill out questionnaires measuring PSF, POF, PJF, and WE. In total, 486 questionnaires were valid. The study found that PSF positively influenced new hires' WE. This positive impact was fully realized through the two mediation paths of POF and PJF. Therefore, a two-mediation model was established. PSF was highly important to the WE of new hires, but this effect was fully realized through the indirect paths of POF and PJF.
\end{abstract}

Keywords: Person-Supervisor fit, Work engagement, Person-Organization fit, Person-Job fit, New hires.

\section{Introduction}

The fit and interaction between people and environment in the workplace has attracted particular attention. Factors include values, personality, aim fit between employees and their organization; consistency of knowledge, skills and ability between employees and their job; the fit between employee and supervisor in terms of values and working style. According to the interactive psychology between human and environment, it is only when people are consistent with the characteristics of the organization (person-organization fit, POF) that they are able to achieve their potential and develop a more positive work attitude and greater efficiency (Baodunwei, 2017). The fit theory also states that person-supervisor fit (PSF) results in a more positive work attitude and better employee behaviors such as work engagement (Marina N Astakhova, 2015). Moreover, person-job fit (PJF) has been significantly correlated with employee's job satisfaction, work engagement(WE) and so on (Alfes, 2017; Gupta, Shaheen, \& Reddy K, 2017).

Work engagement (WE) refers to the positive integration of individuals from physical, cognition and emotion in their work (Wefald A. J., 2009).As a positive and fulfilling working state, WE has been widely recognized for its positive effects on organizational citizenship behavior, happiness, active learning behavior and satisfaction (Hallberg, 2006; Vecina, 2012). For new hires, high level of WE can reduce their depression and turnover intention, help them obtain happiness from work, which plays an important role in transforming them into the role expected by the organization.

Organizations have traditionally recruited new hires as an important way to stimulate individual and organizational innovation and maintain long-term competitiveness (Singh, 2011; Volberda, 2011). And when you're about to bring in a new hire, it's a good idea to gauge their compatibility. This study focuses on new hires and explores the unique role of PSF, POF, PJF on WE. It is theoretical innovation to invoke PSF (a relationship fit perception), PJF (a task-related fit perception), and POF (an environmental perception) in a framework to measure the relationship between person-environment fit and WE.

\section{Literature Review and Hypotheses}

When new hires enter, the organization will implement strategies to help them adapt, including building a bridge for them to communicate with old employees and supervisors, which will help them get more relationship resources from the organization. With relationship resources they can better cope with the negative feelings in the work, improve the sense of work satisfaction, and devote themselves to the work, therefore good PSF has positive influences on new hires' WE. In addition, new hires' work adaptability can be enhanced through training of work knowledge and technical ability. With a better grasp of the skills needed to get the job done and a better understanding of how to do job effectively, new hires can be more positive and abler to feel a sense of accomplishment and fun, their WE will be enhanced naturally. Just as Harter's view "employee engagement occurs when employees know how to possess the resources to complete their works and feel that they play an important role of contribution toward organization" (Harter, 2002). Moreover, building a warm organizational environment and advocating humanistic organizational values can help new hires form organizational identity and sense of belonging, which will promote their work investment level (Guo Yungui, 2016).

Based on the above, hypothesis 1was put forward as follows:

H1: PSF, PJF and POF are positively related to new hires' WE.

New hires need time to learn and adapt to their environment. Their focus is on surviving their new environment and they do not have additional energy to gain a deeper understanding of the organization (Kardkarnklai, 2014). They do not always have a clear understanding of organizational rules and values, which is difficult for them to form a stable understanding. In addition, new hires tend to compare their expectations with the current status of the organization. Some of them can 
integrate into the current organizational environment, while others are unable to reconcile the discrepancy between their expectations and reality. All these can cause them feel unstable within the organization, and POF is relatively low. Therefore, they have to survive in more interactive small groups before they can adapt to the larger organizational environment, in which, leadership plays a critical role. Supervisors, who are passers-by of them, are very familiar with their current mental state and concerns. If supervisors can better guide new hires, the latter can quickly identify with current organization and adapt to their specific role, and early high-quality relationship was associated with enhanced job performance(McKay, 2020). If, however, they fail to receive support from supervisors, their mismatch with the organizational environment will be magnified. New hires' perception of fit with overall company culture predominantly depends on the ways in which their supervisors transmit cultural values within the daily work environment.

Based on the above, hypothesis 2 was put forward:

$\mathrm{H}$ 2: POF mediates the relationship between PSF and new hires' WE.

PJF concerns the demands of employees with regard to their work environment, payment, social status, interpersonal circle, and even professional self-actualization. In Chinese culture, PJF directly affect the new hires' adaptation and learning at work. According to the reciprocity and social exchange theories (Cai, Cai, Sun, \& Ma, 2018), when new hires receive adequate resources and support from their superiors, they return this attention by fully engaging in their work and demonstrating positive emotions. In other words, new hires find their work valuable when their needs are met and the requirements of the job match their own knowledge, skills, and abilities. Thus, supervisors can enhance new hires' working ability by supporting their personal development and providing them with guidance (JOHNSON, 2005). In addition, new hires who receive consistent encouragement can generate enough resources to deal with the requirements of their job and thus find more meaning at work, which can have a positive impact on WE. In conclusion, good PSF can stimulate and promote new hires' PJF, in turn affecting their WE.

Based on the above, hypothesis 3 was put forward:

H3: PJF mediates the relationship between PSF and new hires' WE.

For this study, the researchers constructed a two-mediation model to test the effect of PSF on WE and to investigate the mediating roles of POF and PJF.

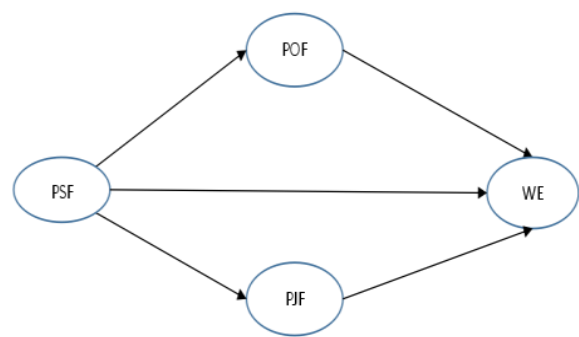

Figure 1: Two-Mediation Model

\section{Materials and Methods}

\subsection{Participants}

The researchers contacted human resources managers from four enterprises in Shandong and Zhejiang provinces via telephone to inform them of the study purpose, and explained that the participants should be informed to answer the questionnaires anonymously and voluntarily. The completed questionnaires were sent back by mail. All identifiable business-related information was removed before processing the data. The survey period lasted six weeks, from October 8 to November 22, 2018. A total of 500 questionnaires were distributed and all of them were returned. However, 14 questionnaires were excluded from the data analysis due to missing data, duplicate data, or random responding, 486 valid questionnaires remained, making the effective recovery rate 96.3\%. The participants included 163 men and 323 women. The average age was 27.61 years $(S D=4.92)$, and the average employment period was 2.74 years $(S D=2.33)$. There were 68 middle managers (4\%), 109 junior managers $(22.4 \%)$, and 309 general employees $(73.6 \%)$.

\subsection{Measurement Instruments}

\subsubsection{Work engagement scale}

The Utrecht work engagement scale (UWES) includes 15 items, such as "I work with enthusiasm", which were classified into three dimensions: vitality, concentration, and dedication (Schaufeli, Salanova, González-romá, \& Bakker, 2002). All items are rated by seven-point scoring, with 0 point (never) being the lowest and 6 points (always) being the highest. The higher the score, the higher the level of work engagement. The structure of UWES was revised in this study, three items with low load were deleted, cronbach's alpha coefficient for the scale was 0.926 .

\subsubsection{Person-organization fit scale and person-job fit scale}

Both the person-organization fit scale and person-job fit scale include six items and are divided into two dimensions with three items each(Cable \& DeRue, 2002), such as "I identify strongly with the goals of my organization", "My abilities and 
training are a good fit with the requirements of my job". In this study, the items were translated by three doctoral students in psychology. Cronbach's alpha coefficients for the the person-organization fit scale was 0.930 and person-job fit scale was 0.899 .

\subsubsection{Person-supervisor fit scale}

The person-supervisor fit scale includes four items, such as "I get along well with my supervisor", which are rated from 1 to 7. (Chuang and Shen, 2007). The structure of the scale was revised in this study, one item with low load was deleted. Cronbach's alpha coefficient of the scale was 0.892 .

\subsection{Data Analysis}

Harman's single-factor test was used to determine whether there was any common method bias by AMOS17.0.

To test the relationship between all of the variables and the mediation hypothesis, strict mediation test procedures were followed. A structural equation model was built using Mplus7.0.

\section{Results}

\subsection{Common Method Bias}

After eliminating the invalid questionnaires, missing value and outlier processing were performed. The common method bias results showed that the fitting index of a four-factor model (this model contains four separate latent variables: POF, PJF, PSF, WE) was better than a single-factor (this model contains only one latent variable, which mixing POF, PJF, PSF, WE together) and two-factor substitution models (this model contains two separate latent variables, POF, PJF, PSF mixing together and WE) (see Table 1). The variance explained by the first factor accounted for $33.16 \%$ in the four-factor model, which was less than the critical threshold of $50 \%$ (Podsakoff et al., 2003). In other words, there was no evidence of common method bias in the results.

Table 1: Comparison of Measurement Models for Common Method Bias

\begin{tabular}{cccccccc}
\hline Model & $\chi^{2}$ & $d f$ & $\chi^{2} / d f$ & CFI & NNFI & GFI & RMSEA \\
\hline Single-factor model & 2287.262 & 358 & 6.389 & 0.756 & 0.771 & 0.753 & 0.504 \\
Two-factor model & 1354.866 & 359 & 3.774 & 0.872 & 0.833 & 0.827 & 0.202 \\
Four-factor model & 792.792 & 364 & 2.178 & 0.923 & 0.911 & 0.956 & 0.060 \\
\hline
\end{tabular}

\subsection{The Control Variables}

In order to avoid the influence of variables other than the independent variables (PSF, POF, PJF) on the outcome variables, the demographic variables were controlled during the correlation analysis and the mediating effect test.

\subsection{Correlation Analysis}

The correlation analysis showed that PSF, POF, PJF, and WE were significantly positively correlated with each other $\left(\mathrm{r}_{\mathrm{PSF} \& \mathrm{POF}}=0.633 * * *, \mathrm{r}_{\mathrm{PSF} \& \mathrm{PJF}}=0.715^{* * *}, \mathrm{r}_{\mathrm{PJF} \& \mathrm{POF}}=0.728 * * *\right.$, $\left.\mathrm{r}_{\mathrm{PSF} \& \mathrm{WE}}=0.608 * * *, \mathrm{r}_{\mathrm{POF} \& \mathrm{WE}}=0.546 * * *, \mathrm{r}_{\mathrm{PJF} \& W E}=0.598 * * *\right)$. The results are calculated based on the sample size of 486 , where: * represents $\mathrm{p}<0.05$, ** represents $\mathrm{p}<0.01$, *** represents $\mathrm{p}<0.001$, the same applies below.

\subsection{Mediation Analysis}

In order to stabilize the mediation effect test results, the bootstrap estimation method (bootstrap $=1,000$ replications) was adopted for data estimation.

First, the researchers used Mplus7.0 to test the mediation role of POF between PSF and WE. The hypothesis model fits ideally $\left(\chi^{2}=619.003, d f=167, p=0.000, \mathrm{CFI}=0.971, \mathrm{TLI}=\right.$ 0.969 , RMSEA $=0.056)$. In this model, the effect of PSF to POF was significant $(\beta=0.686, p<0.001$, CI [0.429, 0.7130]), so as to the effect of POF to WE $(\beta=0.411, p<0.001$; CI $[0.262,0.572])$ and PSF to WE $(\beta=0.289, p<0.001, \mathrm{CI}$ $[0.111,0.353])$. Therefore, POF was found to play a partially mediating role in the relationship between PSF and WE ( $\beta=$ $0.282, p<0.001, \mathrm{CI}[0.179,0.385])$. The mediating effect was $49.39 \%$.

Then, PSF, PJF, and WE were included in the model. The hypotheses model fit perfectly well $\left(\chi^{2}=819.275, d f=167\right.$, $p<0.001, \mathrm{CFI}=0.972$, TLI $=0.955, \mathrm{RMSEA}=0.045)$. In this model, the effects of PSF to PJF was significant $(\beta=0.799, p$ $<0.001$, CI $[0.619,0.885])$, so as to the effect of PJF to WE $(\beta=0.648, p<0.001 ; \mathrm{CI}[0.364,0.797])$ and PSF to WE $(\beta=$ $0.054, \quad p>0.05$; CI $[-0.191,0.200])$. Therefore, PJF played a complete mediating role in the relationship between PSF and WE $(\beta=0.518, p<0.001$, CI $[0.302,0.720])$. The mediating effect was $90.555 \%$.

Finally, by combining all of the assumptions, the overall model fit very well $\left(\chi^{2}=1178.976, d f=293, p<0.001, \mathrm{CFI}=\right.$ 0.971 , TLI $=0.954$, RMSEA $=0.064)$. In this model, there was one direct and two indirect influencing paths to WE. The results showed that the coefficients for both mediating paths were significant, but the coefficient for the direct path was not significant. This indicated that both POF and PJF play a complete mediation role in the effect of PSF on WE. The indirect effect accounted for $93.06 \%$ of the total effect. The details are shown in Table 2 and Figure 2.

Table 2: The Direct and Indirect Effects of Mediation Paths

\begin{tabular}{|c|c|c|c|c|c|}
\hline \multirow{2}{*}{ Effect } & \multirow{2}{*}{ Path } & \multirow[t]{2}{*}{$\begin{array}{l}\text { Standardized } \\
\text { effect values }\end{array}$} & \multirow[t]{2}{*}{$\begin{array}{l}\text { Amount } \\
\text { of effect }\end{array}$} & \multicolumn{2}{|c|}{$\begin{array}{c}\text { Bootstrap 95\% } \\
\text { confidence } \\
\text { interval }\end{array}$} \\
\hline & & & & LLCI & ULCI \\
\hline $\begin{array}{l}\text { Direct } \\
\text { effect }\end{array}$ & PSF $>$ WE & -0.05 & $6.94 \%$ & -0.604 & 0.190 \\
\hline $\begin{array}{l}\text { Indirect } \\
\text { effect }\end{array}$ & $\begin{array}{c}\text { Path1 } \\
\text { PSF }>\text { POF }>\text { WE }\end{array}$ & $0.164^{*}$ & $19.584 \%$ & 0.008 & 0.270 \\
\hline & $\begin{array}{c}\text { Path } 2 \\
\text { PSF }>\text { PJF }>\text { WE }\end{array}$ & $0.506^{*}$ & $19.237 \%$ & 0.191 & 0.998 \\
\hline $\begin{array}{l}\text { Total } \\
\text { indirect } \\
\text { effect }\end{array}$ & & 0.67 & $93.06 \%$ & & \\
\hline $\begin{array}{l}\text { Total } \\
\text { effect }\end{array}$ & & 0.72 & $100 \%$ & & \\
\hline
\end{tabular}




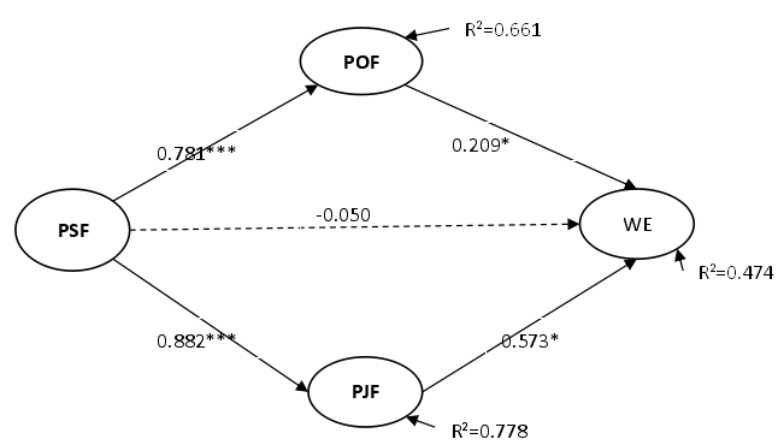

Figure 2: The Mediating Effect of POF and PJF of PSF on WE

\section{Discussion}

The results indicated that PSF, POF,PJF were positively affected WE, which were consistent with the previous findings that POF,PJF,PSF had independent and additive relationship with organizational commitment, job satisfaction (Jang, 2017; Van Vianen, Shen, \& Chuang, 2011). Study of the new teacher also found, first-year teachers' perceptions of POF was the strongest predictor of teacher retention (Miller \& Youngs, 2021). Hoff's paper indicated that job interest fit is strongly related to performance outcomes and satisfaction with one's overall career path.(Hoff, Song, Wee, Phan, \& Rounds, 2020). Schoon (2008) pointed that match between supervisor and subordinate personality dimensions did have strong relationships with the outcome variables of interest (Schoon, 2008). Gupta's (2015) research also revealed that supervisory support could positively affect the work engagement of university teachers. All these well support the conclusion of this study.

This paper also found that both POF and PJF played a mediating role in the process of PSF on WE. This research conclusion was also supported by many studies. Positive PSF encourages employees to view all aspects of the organizational environment from a positive perspective, they also receive higher-quality resources (such as better office equipment) and encouragement. The relationship with supervisors not only has an effect on the pressure that employees feel, but also the work input of them through their intermediary role(Dinh, 2019). Marina's paper stated that in the U.S., the relationships of PSF on OC are fully transmitted through POF, whereas, in Japan, both direct and indirect links are supported.(Marina N. Astakhova, 2016). Jang have identified that individual's perceptions of fit with their supervisors were important in strengthening or weakening the relationships between POF PJF and OC (Jang, 2017). Moreover, according to the theory of human-environment interaction, if employees feel positive about their initial interactions with their environment (i.e. PSF and PJF), they are more likely to form a positive action model for the next round of interactions. Effective and deep-rooted behaviors are formed (e.g. WE). However, if the employee encounters setbacks in the initial stages, they are exposed to stress and other forms of psychological anxiety, especially in complex situations in which they are moving from the initial behavior to the effective behavior phase. At this time, external support is needed to resist setbacks and find a way to form effective behaviors. Thus, the role of supervisor becomes more important; they are likely to understand the feelings of pressure, anxiety, and other negative emotions that the new hires might be experiencing, and might know how to guide them to better overcome difficulties. Therefore, the supervisor acts as a bridge between the employee and different elements of their external environmental (e.g. organizational environment characteristics, organizational values, work characteristics, team member conflicts), so that the initial behavior can be effectively extended to the stage of effective behavior (i.e. WE), and the deficiencies of the initial behavior can be improved. Overall, adequate PSF helps new hires strive for more intangible resources (such as encouragement), and further affects their WE through the mediating influences of POF and PJF.

\section{Contributions}

First, from the perspective of the interaction between person and environment, this study explores the internal influence mechanism of multiple fit factors on new hires' WE, which compensates for the knowledge gap on the influence of single fit factor on employee's behavior and provide a more comprehensive picture about the influence of PEF perceptions. Previous research has emphasized the importance of exploring the mechanisms of interaction between matching factors from an interaction perspective (Kristof-Brown, Zimmerman, \& Johnson, 2005). Bronfenbrenner's ecosystem theory also emphasizes the individual's existence within a multi-level system; the different levels of this system impact differently on human development, and they effect each other mutually. For employees, behavior is not only influenced by job characteristics, workplace, and the relationships between colleagues, but also by organizational values and vision. Therefore, to invoke PSF, POF, PJF into one study and explore their mutual influence have unique contribution.

Second, this paper stressed the role of PSF in the three kinds of fit perceptions. New hires typically require considerable energy to adapt to their new job and role change, they have little time to explore the organizational atmosphere. The interaction between them and their supervisor is an important factor that affects their perception of organization and job, which indicates that relationships comprise the most important organizational factor. Among all interpersonal relationships at work, the relationship between employees and their immediate supervisor is the most important. The subordinate-superior relationship is a reward and punishment mechanism for employees, which must arouse the management practice department's attention.

\section{Implications}

Recruiting is the first step for companies. During the recruiting process, candidates who are not good fit with the organizational culture and jobwhould be passed over. Once employees are recruited, the company will take many measures to help them adapt as soon as possible and tap to their potential and make contributions. Among which, the interpersonal training (such as respecting others, treating others with sincerity, teamwork) enables the relationship 
between new hires and supervisor to be improved. Moreover, the implementation of organizational cultural activities can help to improve organizational cohesion. Training in job responsibilities, procedures, standards could help new hires integrate into the workforce more quickly. In this way, the matching of relationship with supervisors, skills with job, and recognition with the organization influences new hires' loyalty and investment.

Moreover, this paper emphasizes the fit between new hires and their supervisors, which puts forward high requirements for supervisors. On one hand, in China leaders are expected to inspire awe in their subordinates to achieve the so-called power distance. On the other hand, they should also be charismatic leaders to meet the needs of every team member, of course their skills and knowledge should be subdued by their subordinates.

\section{Limitations and Future Research Directions}

First, this study relied on cross-sectional data. Although the research design was based on existing theories, only limited conclusions can be drawn about causality. Future studies may involve constructing intervention and control groups through longitudinal designs.

Secondly, this study only discusses the influence of PSF, POF, PJF on employee's WE. However, there are additional factors that may influence WE, such as person-vocation fit (the fit that human personality characteristics are consistent with the professional nature), person-group fit (the consistency with other team members and leaders in personality and other aspects). Future research could expand on these variables and their relationships with WE.

\section{Acknowledgement}

This research was supported by Shandong Province social science program for Youth (20DJYD07) and Shandong University Education Reform Research Project(M2020251).

\section{References}

[1] Alfes, K. F., Nils. (2017). Paradoxical leadership: Understanding and managing conflicting tensions to foster volunteer engagement. Organizational Dynamics, 46(2), 96-103. doi:10.1016/j.orgdyn.2017.04.00

[2] Astakhova, M. N. (2015). Person-Supervisor and Person-Organization Fit and Organizational Commitment in the U.S. and Japan. academy of management, 1, 2151-6561. doi:https://doi.org/10.5465/ambpp.2015.10670abstract

[3] Astakhova, M. N. (2016). Explaining the effects of perceived person-supervisor fit and person-organization fit on organizational commitment in the U.S. and Japan. Journal of Business Research, 69(2), 956-963. Retrieved from

http://www.sciencedirect.com/science/article/pii/S0148 296315003884.

doi:https://doi.org/10.1016/j.jbusres.2015.08.039
[4] Baodunwei. (2017). The effect of person organization fit, person group fit, person job fit to the performance of employees: the mediation role of emotion commitment. (Doctor), Shanghai International Studies University, Shanghai.

[5] Cable, D., \& DeRue, D. (2002). The Convergent and Discriminant Validity of Subjective Fit Perceptions. The Journal of applied psychology, 87, 875-884. doi:10.1037//0021-9010.87.5.875

[6] Cai, D., Cai, Y., Sun, Y., \& Ma, J. (2018). Linking Empowering Leadership and Employee Work Engagement: The Effects of Person-Job Fit, Person-Group Fit, and Proactive Personality. Frontiers in Psychology, 9. doi:10.3389/fpsyg.2018.01304

[7] Dinh, L. N. (2019). Determinants of employee engagement mediated by work-life balance and work stress. Management Science Letters, 4, 923-928. doi:10.5267/j.msl.2019.10.003

[8] Guo Yungui, z. 1. (2016). Research on the influence mechanism of organizational socialization on job engagement - from the perspective of identity theory. $\begin{array}{lll}\text { Soft } & \text { science, } & 30(4),\end{array}$ doi:10.13956/j.ss.1001-8409.2016.04.15

[9] Gupta, M., Shaheen, M., \& Reddy K, P. (2017). Impact of Psychological Capital on Organizational Citizenship Behavior: Mediation by Work Engagement. Journal of Management Development, 36. doi:10.1108/JMD-06-2016-0084

[10] Hallberg, U. E. a. S., W.B. (2006). "Same Same" but Different? Can Work Engagement Be Discriminated from Job Involvement and Organizational Commitment? European Psychologist, 11, 119-127. doi:http://dx.doi.org/10.1027/1016-9040.11.2.119

[11] Harter, J. K., Schmidt, Frank L., Hayes, Theodore L. (2002). Business-unit-level relationship between employee satisfaction, employee engagement, and business outcomes: A meta-analysis. Journal of Applied Psychology, $\quad 87(2), \quad 268-279$ doi:10.1037//0021-9010.87.2.268

[12] Hoff, K., Song, Q. C., Wee, C., Phan, W. M. J., \& Rounds, J. (2020). Interest Fit and Job Satisfaction: A Systematic Review and Meta-Analysis. Journal of Vocational Behavior. doi:10.1016/j.jvb.2020.103503

[13] Jang, S. L. a. E. (2017). The Relationships of Person-Organization Fit and Person-Job Fit with Work Attitudes: A Moderating Effect of Person-Supervisor Fit. Journal of Engineering and Applied Sciences, 12(14), 3767-3778. doi:10.36478/jeasci.2017.3767.3778

[14] Johnson, A. L. K. B. R. D. Z. E. C. (2005). CONSEQUENCES OF INDIVIDUALS' FIT AT WORK: A META - ANALYSIS OF PERSON - JOB, PERSON - ORGANIZATION, PERSON - GROUP, AND PERSON - SUPERVISOR FIT. Personal Psychology, 58(2), 281-342. doi:https://doi.org/10.1111/j.1744-6570.2005.00672

[15] Kardkarnklai, U. m. (2014). Organizational Culture: The Perspectives of New Hires and Existing Employees of a Pharmaceutical Company in Thailand. Asian Social Science, 10. doi:10.5539/ass.v10n14p224

[16] Kristof-Brown, A., Zimmerman, R., \& Johnson, E. (2005). Consequences of Individual's Fit at Work: A Meta-Analysis of Person-Job, Person-Organization, Person-Group, and Person-Supervisor Fit. Personnel 
Psychology, 58, 281-342. doi:10.1111/j.1744-6570.2005.00672.x

[17] McKay, L. Z. C. B. G. P. F. (2020). From new hires to their supervisors: The influence of newcomer race/ethnicity on the leader-member exchange conveyance. Journal of Occupational and Organizational Psychology, 93(3), 767-789. doi:https://doi.org/10.1111/joop.12314

[18] Miller, J., \& Youngs, P. (2021). Person-organization fit and first-year teacher retention in the United States. Teaching and Teacher Education, 97, 103226. doi:10.1016/j.tate.2020.103226

[19] Schaufeli, W. B., Salanova, M., González-romá, V., \& Bakker, A. B. (2002). The Measurement of Engagement and Burnout: A Two Sample Confirmatory Factor Analytic Approach. Journal of Happiness Studies, 3(1), 71-92. Retrieved from https://doi.org/10.1023/A:1015630930326. doi:10.1023/A:1015630930326

[20] Schoon, H. J. (2008). PERSON-SUPERVISOR FIT: IMPLICATIONS FOR ORGANIZATIONAL STRESS,

[21] ORGANIZATIONAL COMMITMENT, AND JOB SATISFACTION. Clemson University TigerPrints, 6 . doi:https://tigerprints.clemson.edu/all_theses

[22] Singh, J. (2011). Recruiting for Ideas: How Firms Exploit the Prior inventions of New Hires. management science, 57(1), 129-150. doi:10.1287/mnsc.1100.1253

[23] Van Vianen, A., Shen, C.-T., \& Chuang, A. (2011). Person-organization and person-supervisor fits: Employee commitments in a Chinese context. Journal of Organizational Behavior, 32, 906-926. doi:10.1002/job.726

[24] Vecina, M. (2012). Volunteer engagement: Does engagement predict the degree of satisfaction among new volunteers and the commitment of those who have been Active Longer?. Applied Psychology, 61(1), 130-148. doi:10.1111/j1464-0597.2011.00460.x

[25] Volberda, H. W., Morgan, Robert E., Reinmoeller, Patrick, Hitt, Michael A., Ireland, R. Duane and Hoskisson, Robert E. (2011). Cengage Learning.

[26] Wefald A. J., D. R. G. (2009). Construct dimensionality of engagement and its relation with satisfaction. The Journal of Psychology, 143(1), 91-111. doi:10.3200/JRLP.143.1.91-112. PMID: 19157075 